\title{
Estrutura da comunidade de macroinvertebrados associados ao folhiço submerso de remanso e correnteza em igarapés da Amazônia Central
}

\author{
Carla Ferreira Rezende \\ Biota Neotropica v7 (n2) \\ http://www.biotaneotropica.org.br/v7n2/pt/abstract?short-communication+bn01607022007 \\ Recebido em 08/11/06 \\ Versão reformulada recebida em 20/03/07 \\ Publicado em 01/05/07 \\ Programa de Pós Graduação em Ecologia, Laboratório de Ecologia de Peixes, \\ Departamento de Ecologia, Instituto de Biologia, Universidade Federal do Rio de Janeiro - UFRJ \\ Av. Mal. Trompowski, s/n, CCS Bloco A, CP 68020, CEP 21941-590, Rio de Janeiro, RJ, Brasil \\ Autor para correspondência: Carla Ferreira Rezende, e-mail: carla.fr@terra.com.br, \\ http://www.ppgeco.biologia.ufrj.br/
}

\begin{abstract}
Rezende, C.F. Community structure of macroinvertebrates associated with leaf litter from riffles and pools from Central Amazon streams. Biota Neotrop. May/Aug 2007 vol. 7, no. 2 http://www.biotaneotropica. org.br/v7n2/pt/abstract?short-communication+bn01607022007 ISSN 1676-0603.

Considering that the composition and distribution of freshwater macroinvertebrates is related to several environmental factors, especially water flow and type of substratum I hypothesized that composition, richness and abundance of macroinvertebrates that occur in both environments with distinct hydrological characteristics (riffle and pool) were different between these areas. Specimens were sampled in three streams where five traps were arranged in stretches from pools and riffles totalizing 30 traps. In the laboratory the macroinvertebrates were identified to family level. An analysis of variance in blocks was made in order to verify possible effects of habitat and stream type on the richness and abundance of macroinvertebrates. Shannon's diversity index was applied, followed by a $t$-test. The Bray-Curtis cluster analysis was performed to verify the similarity between different habitats. A total of 132 specimens were collected, 97 in riffles and 35 in pools. Among sixteen families collected, six colonized only riffles, two colonized only pools and eight colonized both environments. An effect of the habitat (pools or riffles) upon the richness and abundance of macroinvertebrates was detected. On the contrary, no difference was found among streams (igarapés). The Shannon diversity index was higher in riffles than in pools. The riffle stretches formed a single group on the Bray-Curtis cluster analysis. I conclude that composition and abundance were distinct between the community of macroinvertebrates of riffles and pools. The explaining hypothesis for these results is that the community structure of both environments is distinct due to variations associated with water flow, that allows the existence of groups with different ecological and behavioral features.

Keywords: colonization, water flow, aquatic invertebrates.

\section{Resumo}

Rezende, C.F. Estrutura da comunidade de macroinvertebrados associados ao folhiço submerso de remanso e correnteza em igarapés da Amazônia Central. Biota Neotrop. May/Aug 2007 vol. 7, no. 2 http:// www.biotaneotropica.org.br/v7n2/pt/abstract?short-communication+bn01607022007 ISSN 1676-0603.

Considerando que a composição e a distribuição de macroinvertebrados de água doce é relacionada a diversos fatores ambientais, destacando-se a velocidade da corrente e o tipo de substrato tive como hipótese de trabalho que a composição, riqueza, e abundância de macroinvertebrados que ocorrem em dois ambientes com características hidrológicas distintas (correnteza e remanso) fossem diferentes entre essas áreas. A amostragem foi realizada em três igarapés onde foram dispostas cinco armadilhas em trechos de remanso e cinco em correnteza, totalizando 30 armadilhas. No laboratório os macroinvertebrados foram identificados em ordens e os indivíduos de cada ordem foram separados em famílias. Análise de variância em blocos foi realizada com intuito de verificar possíveis efeitos do tipo de hábitat e dos rios sobre riqueza e abundância de macroinvertebrados. O índice de diversidade Shannon foi aplicado, seguido do teste $t$. Análise de agrupamento (UPGMA) usando o coeficiente de distância de BrayCurtis foi realizada com intuito de verificar a similaridade entre os diferentes hábitats dos rios. Foram coletados 132 especimens, 97 em correnteza e 35 em remanso. Dentre as dezesseis famílias coletadas, seis colonizaram apenas correnteza, dois apenas remanso e oito colonizaram os dois ambientes. Foi detectado um efeito do hábitat (remanso ou correnteza) sobre a riqueza e abundância de macroinvertebrados. No entanto, não foi detectado efeito do local (igarapés). A diversidade de Shannon foi maior para correnteza do que para o remanso. A análise de agrupamento de Bray-Curtis formou um agrupamento mais definido entre os trechos de corredeira. Concluo que a composição e a abundância são os parâmetros que diferem entre as comunidades de macroinvertebrados
\end{abstract}


de correnteza e remanso. A hipótese explicativa para este resultado é de que a estrutura da comunidade desses dois ambientes se diferencie devido às variações associadas ao fluxo da correnteza, que possibilitam a existência de grupos com características ecológicas e comportamentais distintas nos dois ambientes.

Palavras-chave: colonização, velocidade de correnteza, invertebrados aquáticos.

\section{Introdução}

Dentre os hábitats da Amazônia central destacam-se as florestas de terra-firme, que se caracterizam por apresentarem pequenos riachos não submetidos a pulsos de inundação. Esses sistemas são submetidos a chuvas locais em menor amplitude, porém em maior freqüência (Junk et al. 1989). Estes riachos, conhecidos localmente como igarapés, são importantes componentes das florestas tropicais e criam uma heterogeneidade estrutural característica no ecossistema de terra-firme (Mortati 2004).

Uma característica dos igarapés de terra-firme até terceira ordem é que o denso dossel da vegetação marginal diminui a produção primária autóctone devido ao sombreamento da área. As folhas que caem na água se depositam no fundo do igarapé contribuindo para a formação de microhabitats que são utilizados como abrigo e alimento para a fauna aquática (Vannote et al. 1980, Hirabayashi \& Wotton 1998, Passos et al. 2003).

A composição e a distribuição espacial da fauna de macroinvertebrados de água doce está relacionada a diversos fatores ambientais, destacando-se a velocidade da corrente e o tipo de substrato (Hynes 1970, Allan 1995, Nemeth 1998). As variações na velocidade da correnteza aumentam a diversidade desses organismos, influenciando na distribuição do alimento, remoção de nutrientes e disponibilidade de microhabitats (Merritt \& Cummins 1984, Allan 1995). Já o tipo de substrato se relaciona com a composição dos organismos, sendo o determinante na distribuição e abundância dos espécimes associados (Minshall 1984).

Os substratos inorgânicos possuem como características determinantes de sua colonização o tamanho e a superfície. Partículas menores como areia e silte são muito instáveis podendo ser carreadas rio a baixo; pedras e matacões são mais estáveis permanecendo fixas mesmo quando expostas a velocidade de correntezas elevadas (Allan 1995). Partículas orgânicas menores do que $1 \mathrm{~mm}$ normalmente servem como alimento, poucas exceções são encontradas sendo utilizadas para a fixação de invertebrados muito pequenos e microorganismos (Allan 1995, Afonso \& Henry 2002). O material orgânico proveniente da vegetação ripária e marginal funciona como substrato submerso para fixação dos organismos, sendo extremamente utilizados por fornecerem abrigo e alimento (Uieda \& Gajardo 1996), visto que a deposição de detritos e a colonização por algas ocorre em pouco tempo neste tipo de substrato (Allan 1995).

Dentre os substratos comumente colonizados por macroinvertebrados, pedras grandes constituem o substrato com maior diversidade (Allan 1995) e areia o mais pobre contendo poucas espécies (Kikuchi \& Uieda 2005). Já o folhiço sustenta uma grande diversidade e abundância de invertebrados (Allan 1995, Kikuchi \& Uieda 2005), sendo um substrato interessante para estudos de curta duração.

A determinação do papel do substrato para a comunidade de macroinvertebrados é uma tarefa complicada, porque os substratos inevitavelmente estão associados com as variáveis ambientais. A velocidade da correnteza juntamente com variáveis físicas associadas ao fluxo da água representa o fator ambiental mais importante para os organismos de riachos (Allan 1995).

A partir destas considerações escolhi o substrato orgânico "folhiço", submetido a duas condições ambientais diferenciadas pela velocidade da correnteza para testar o objetivo e a hipótese a seguir: $\mathrm{O}$ objetivo foi comparar a estrutura da comunidade de ma- croinvertebrados através da colonização do folhiço submerso em duas áreas de igarapé, uma área de remanso e outra de correnteza. A hipótese era de que composição, riqueza e abundância de espécies de macroinvertebrados fossem diferentes entre as áreas.

\section{Área de estudo}

O projeto foi desenvolvido na Fazenda Esteio, em três igarapés que cortam a trilha Q da Reserva do $\mathrm{km} \mathrm{41.} \mathrm{Esta} \mathrm{é} \mathrm{uma} \mathrm{reserva} \mathrm{de}$ terra firme de 1000 ha situada a cerca de $80 \mathrm{~km}$ ao norte de Manaus, $\mathrm{AM}\left(02^{\circ} 28^{\prime} \mathrm{S}\right.$ e $\left.59^{\circ} 46^{\prime} \mathrm{O}\right)$, onde são encontrados diversos igarapés de primeira e segunda ordem (http://pdbff.inpa.gov.br/esteio.html, acesso 16/02/2007).

As florestas de terra-firme são divididas em três diferentes mesohábitats definidos principalmente pelo tipo de solo e relevo: platô, vertente e baixio. Nas áreas de baixio encontram-se os igarapés. As áreas de terra-firme não estão sujeitas ao pulso de inundação, sendo as variações pluviais são ocasionadas pelas chuvas locais (Junk et al. 1989).

Esses igarapés são caracterizados por possuírem cobertura de dossel bastante densa e substrato predominantemente arenoso, intercalado, por extensos bancos de folhiço e troncos caídos.

\section{Material e Métodos}

A amostragem foi realizada em três igarapés de $1^{\mathrm{a}}$ ordem, em trechos de remanso e correnteza. Considerei áreas de remanso como meandros de água praticamente parada e correnteza como trechos onde a velocidade da água era superior a $20 \mathrm{~cm} / \mathrm{s}$ (Mortati 2004). Em cada um dos três igarapés foram aleatoriamente dispostas cinco armadilhas em trechos de remanso e cinco em correnteza, totalizando 30 armadilhas. As armadilhas consistiam em sacos de nylon com malha de aproximadamente de $20 \mathrm{~mm}$ entre nós adjacentes. Os sacos tinham $40 \mathrm{~cm}$ de comprimento por $15 \mathrm{~cm}$ de diâmetro, foram fechados nas duas extremidades e presos por estacas no fundo do igarapé, em áreas de remanso e correnteza. Estes sacos foram preenchidos com $80 \mathrm{~g}$ de folhiço retirado do igarapé e lavado com intuito retirar qualquer animal que estivesse fixado ao substrato. Folhas não decompostas foram selecionadas para compor as armadilhas. Após 48 horas as armadilhas foram recolhidas e acondicionadas individualmente em sacos plásticos etiquetados. No laboratório os macroinvertebrados foram identificados de acordo com suas respectivas ordens e os indivíduos de cada ordem foram separados em famílias.

Análise de variância em blocos (ou pareada) foi realizada a fim de se verificar possíveis efeitos do tipo de hábitat (remanso ou correnteza) e dos rios sobre riqueza e abundância de macroinvertebrados. Os rios foram analisados como um fator "bloco" para minimizar possíveis variações entre as amostras dentro de cada rio. O fator "bloco" avalia a variação entre os grupos (rios) minimizando os efeitos dentro de cada grupo (amostras) (Zar 1999).

O índice de diversidade Shannon (Krebs 1999) foi aplicado, seguido do teste $t$ (Zar 1999) para verificar possíveis diferenças entre a diversidade das duas áreas. Análise de agrupamento (UPGMA) usando o coeficiente de distância de Bray-Curtis (Krebs 1989) foi realizada com intuito de verificar a similaridade entre os diferentes hábitats dos rios (remanso e correnteza). 


\section{Resultados}

Foram coletados no total 132 espécimens, 97 em correnteza e 35 em remanso, distribuídas entre duas classes, oito ordens e dezesseis famílias. Dentre as dezesseis famílias seis, colonizaram apenas correnteza, dois apenas remanso e oito colonizaram os dois ambientes (Tabela 1). A composição dos organismos mais abundantes diferiu entre os hábitats corredeira e remanso. $\mathrm{Na}$ corredeira foi encontrado um predomínio de Trichoptera (31) seguido por Plecoptera (23) e Diptera (14) e no remanso houve um predomínio de Trichoptera (13) seguido por Decapoda (9) e Ephemeroptera (7).

As riquezas e abundâncias médias dos três rios foram maiores nas áreas com correnteza do que nas áreas de remanso (Figura 1 e 2).

Foi detectado um efeito do hábitat (remanso ou correnteza) sobre a riqueza de famílias $(\mathrm{F}=8,42 ; \mathrm{p}=0,009 ; \mathrm{gL}=1)$ e abundância $(\mathrm{F}=9,35 ; \mathrm{p}=0,006 ; \mathrm{gL}=1)$ de macroinvertebrados. No entanto, não foi detectado efeito do local (igarapés) sobre a riqueza $(\mathrm{F}=0,69$; $\mathrm{p}=0,51 ; \mathrm{gL}=2)$ e nem sobre a abundância $(\mathrm{F}=0,88 ; \mathrm{p}=0,42$; $\mathrm{gL}=2$ ) (Figura 1 e 2).

A diversidade de Shannon foi de 2,18 para correnteza e de 2,02 para o remanso, não diferindo significativamente $(\mathrm{t}=-1,017$; $\mathrm{p}=0,31)$.
A análise de agrupamento (UPGMA) usando o coeficiente de distância de Bray-Curtis formou um agrupamento entre os trechos de correnteza, demonstrando maior similaridade entre estes trechos,

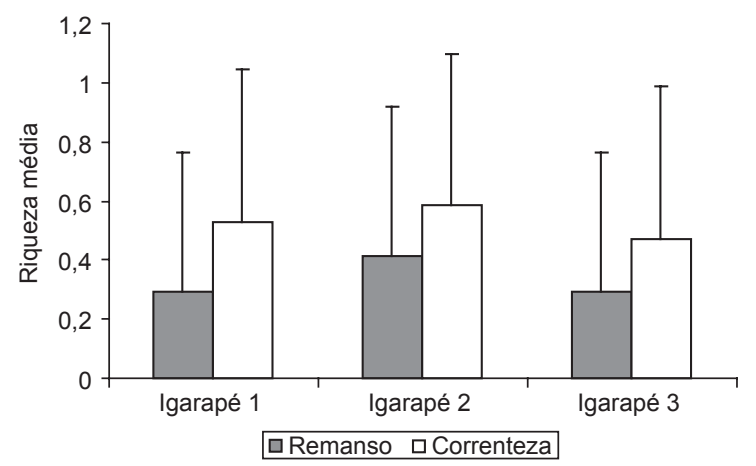

Figura 1. Média e desvio padrão da riqueza de famílias de macroinvertebrados em trechos de corredeira e remanso, em três igarapés na Reserva do km 41, Amazônia central.

Figure 1. Mean and Standard Deviation in family richness in pool and riffle stretches of three streams at km 41, Central Amazon.

Tabela 1. Abundância de famílias dos artrópodes encontrados nas armadilhas de folhiço submerso dos três igarapés estudados da reserva do km 41, Amazônia central.

Table 1. Abundance from family of arthropod families from leaf litter found in three streams at km 41, Central Amazon.

\begin{tabular}{|c|c|c|c|c|c|c|c|}
\hline \multirow[t]{3}{*}{ Táxon } & \multicolumn{6}{|c|}{ Local } & \multirow[t]{3}{*}{ Total } \\
\hline & \multicolumn{2}{|c|}{ Igarapé 1} & \multicolumn{2}{|c|}{ Igarapé 2} & \multicolumn{2}{|c|}{ Igarapé 3} & \\
\hline & Remanso & Correnteza & Remanso & Correnteza & Remanso & Correnteza & \\
\hline \multicolumn{8}{|l|}{ Decapoda } \\
\hline Palaemonidae & 0 & 0 & 8 & 0 & 1 & 1 & 10 \\
\hline \multicolumn{8}{|l|}{ Diptera } \\
\hline Simuliidae & 0 & 0 & 0 & 14 & 0 & 0 & 14 \\
\hline \multicolumn{8}{|l|}{ Ephemeroptera } \\
\hline não identificado & 1 & 1 & 0 & 7 & 0 & 1 & 10 \\
\hline Polymitarcyidae & 3 & 0 & 2 & 2 & 1 & 0 & 8 \\
\hline Baetidae & 0 & 0 & 0 & 0 & 0 & 1 & 1 \\
\hline \multicolumn{8}{|l|}{ Plecoptera } \\
\hline Perlidae & 0 & 13 & 1 & 9 & 2 & 1 & 26 \\
\hline \multicolumn{8}{|l|}{ Megaloptera } \\
\hline Corydalidae & 0 & 0 & 0 & 2 & 0 & 0 & 2 \\
\hline \multicolumn{8}{|l|}{ Coleoptera } \\
\hline Elmidae - adulto & 0 & 2 & 0 & 2 & 2 & 0 & 6 \\
\hline Limnichidae & 0 & 3 & 0 & 1 & 0 & 0 & 4 \\
\hline Elmidae - larva & 0 & 1 & 0 & 0 & 0 & 1 & 2 \\
\hline \multicolumn{8}{|l|}{ Odonata } \\
\hline Polythoridae & 0 & 0 & 0 & 2 & 0 & 0 & 2 \\
\hline Libellulidae & 0 & 1 & 0 & 1 & 1 & 0 & 3 \\
\hline \multicolumn{8}{|l|}{ Trichoptera } \\
\hline Calamoceratidae & 0 & 5 & 3 & 0 & 0 & 5 & 13 \\
\hline Hydrobiosidae & 0 & 0 & 1 & 0 & 0 & 0 & 1 \\
\hline Leptoceridae & 2 & 0 & 0 & 0 & 0 & 0 & 2 \\
\hline Helicopsychidae & 2 & 1 & 1 & 0 & 0 & 2 & 6 \\
\hline Hydropsychidae & 3 & 8 & 1 & 4 & 0 & 6 & 22 \\
\hline Total & 11 & 35 & 17 & 44 & 7 & 18 & 132 \\
\hline
\end{tabular}


do que em relação aos trechos de remanso, onde somente dois deles (R1 e R2) formaram um agrupamento (Figura 3).

\section{Discussão}

A colonização em áreas de correnteza e remanso diferiu quanto à riqueza e à abundância de macroinvertebrados, sendo ambas maiores na correnteza; outros trabalhos na região neotropical corroboram o padrão encontrado (Salcedo \& Fonseca 1998, Kikuchi \& Uieda 2005). Segundo Greenwood \& Richard-Coulet (1996) os ambientes de correnteza e remanso são contrastantes e proporcionam a fixação de faunas características, sendo observada a preferência de alguns táxons.

Maior riqueza e abundância de espécies de macroinvertebrados em áreas de correnteza são amplamente discutidas na literatura (e.g. Allan 1995, Uieda \& Gajardo 1996, Nemeth 1998, Kikuchi \& Uieda 2005). Desta forma, duas hipóteses podem explicar a maior colonização em hábitats de correnteza. 1) este ambiente possui

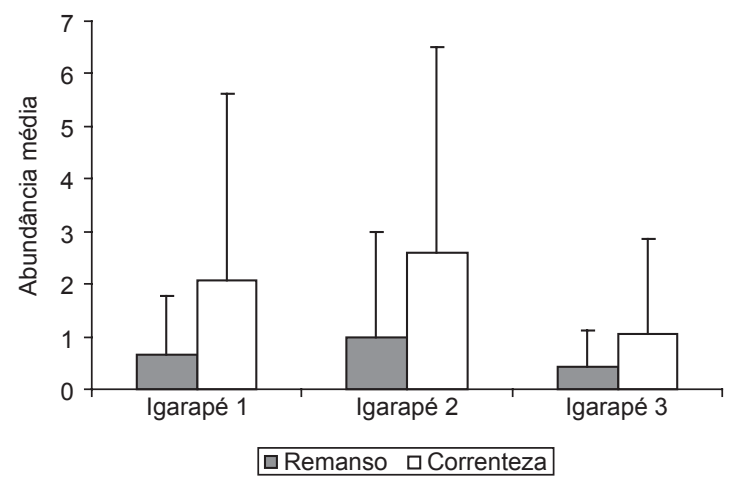

Figura 2. Média e desvio padrão de abundância de macroinvertebrados em trechos de corredeira e remanso, em três igarapés na Reserva do km 41, Amazônia central.

Figure 2. Mean and Standard Deviation in abundance of macroinvertebrates in pool and riffle stretches of three streams at in $\mathrm{km} \mathrm{41,} \mathrm{Central} \mathrm{Amazon.}$

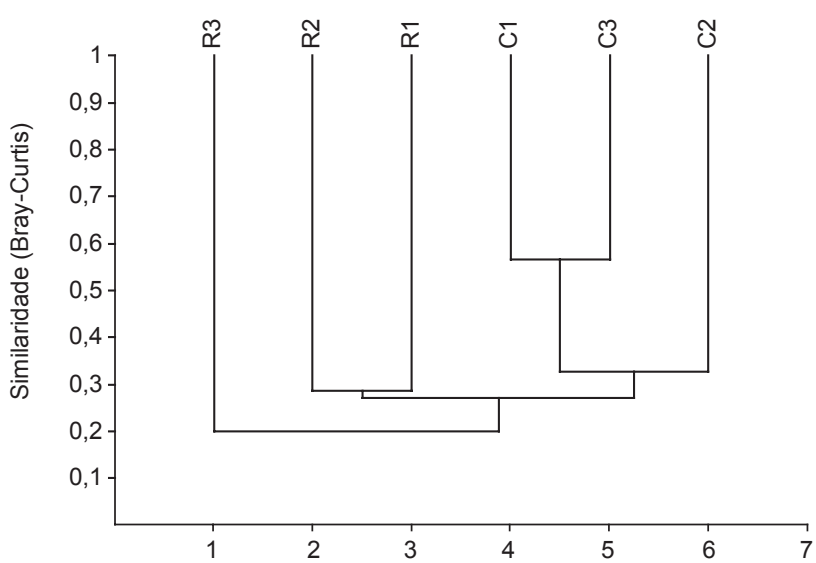

Figura 3. Análise de agrupamento (Bray-Curtis) para habitats de remanso (R1- Remanso Igarapé 1, R2 - Remanso do Igarapé 2, R3 - Remanso do Igarapé 3) de corredeira (C1 - Corredeira do Igarapé 1, C2 - Corredeira do Igarapé 2, C3 - Corredeira do Igarapé 3) e dos três igarapés da reserva do km 41, Amazônia central.

Figure 3. Cluster analysis (UPGMA, Bray-Curtis) for pool (R1- pool stream 1, $\mathrm{R} 2$ - pool stream 2, R3 - pool stream 3) and for riffle habitats (C1- riflle stream 1, C2 riffle stream 2, C3 riffle stream 3) for three streams at $\mathrm{km} \mathrm{41,}$ Central Amazon. maior riqueza e abundancia devido a fatores como disponibilidade de oxigênio e alimento; 2) a colonização mais rápida neste ambiente ocorre devido ao processo de deriva, onde os organismos aquáticos se desprendem do substrato ao qual estavam fixados e se fixam em um novo substrato correnteza abaixo (Merritt \& Cummins 1984).

Os resultados apontam diferenças de composição entre os ambientes. A presença de grupos característicos de correnteza e remanso vem sendo discutida na literatura. O predomínio de Trichoptera (Calamoceratidae e Hydropsychidae), Plecoptera (Perlidae) e Diptera (Simuliidae) em ambientes de correnteza havia sido encontrado anteriormente por Scullion et al. (1982); a abundância destes grupos em correnteza também é discutida por Greenwood \& Richard-Coulet (1996) corroborando o padrão encontrado. Aspectos comportamentais e ecológicos podem justificar essas predominâncias. Plecópteros e dípteros (principalmente Simuliidae) apresentam, respectivamente, adaptações morfológicas direcionadas à utilização do oxigênio dissolvido e captura de alimento relacionado aos ambientes com correnteza (Pennak 1978, Merritt \& Cummins 1984) o que pode justificar a maior abundância destas duas ordens nesses ambientes.

A diversidade de Shannon não difere entre os dois ambientes. Desta forma, concluo que a composição e a abundância são os parâmetros que diferem entre as comunidades de macroinvertebrados de folhiço de correnteza e remanso. A hipótese explicativa para este resultado é de que a estrutura da comunidade desses dois ambientes se diferencie devido às variações associadas ao fluxo da correnteza, que possibilitam a existência de grupos com características ecológicas e comportamentais distintas nos dois ambientes.

\section{Agradecimentos}

Este trabalho foi realizado durante o Curso de Campo Ecologia da Floresta Amazônica, versão 2005, oferecido pelo Instituto Nacional de Pesquisas da Amazônia/ Projeto Dinâmica Biológica de Fragmentos Florestais (INPA/PDBFF). Agradeço ao CNPQ (140928/2005-7) pela bolsa concedida. Agradeço ao Juruna e ao Victor Trivério Cardoso pelo auxílio no campo, Adalberto J. Santos pela ajuda na identificação do material, Henrique Nascimento e Glauco Machado pelas sugestões durante o curso de campo, Jorge Nessimian e Cristiane HenriquesOliveira pelas sugestões do trabalho após o curso de campo. A Rosana Mazzoni e Érica Pellegrini Caramaschi pela leitura do manuscrito; Miriam Pliz Albrecht pela correção do abstract e a todos os amigos do curso de campo EFA que participaram da versão 2005.

\section{Referências Bibliográficas}

AFONSO, A.A. \& HENRY, R. 2002. Retention of particulate organic matter in tropical headstream. Hydrobiologia. 482: 161-166.

ALLAN, J.D. 1995. Stream ecology. Kluger Academic Press, U.S.A.

GREENWOOD, M.T. \& RICHARD-COULET, M. 1996. Aquatic invertebrates. In Fluvial Hydrosystems (G.E. Petts \& C. Amoros, eds). Chapman \& Hall, London, p. 137-164.

HIRABAYASHI, K. \& WOTTON, R.S. 1998. Organic matter processing by chironomid larvae (Diptera: Chironomidae). Hydrobiologia. 382: 151-159.

HYNES, H.B.N. 1970. The ecology of running waters. 3 ed. Canada Toronto Press, Canada.

JUNK, W.J., BAYLEY, P.B. \& SPARKS, R.E. 1989. The flood pulse concept in river-floodplain systems. In Proceedings of the international large river symposium (P.D. Dodge, ed). Can. Spec. Publ. Fish. Aquat. Sci., 106:110-127.

KIKUCHI, R. M. \& UIEDA, V.S. 2005. Composição e distribuição dos macroinvertebrados em diferentes substratos de fundo de um riacho no município de Itatinga, São Paulo, Brasil. Entomol. Vect. 12(2): 193-231. 
KREBS, C.J. 1989. Ecological metodology. Harper \& Hall, New York, US.

KREBS, C.J. 1999. Ecological Methodology. Harper Collins Publishers, New York.

MERRITT, R.W. \& CUMMINS, K.W. 1984. An introduction to the aquatic insects of North America. Kendall, Hunt Publication Co., Dubuque, Iowa.

MINSHALL, G.W. 1984. Aquatic insect-substratum relationships. The ecology of Aquatic Insects (V.H. Resh \& D.M. Rosenberg, eds). Praeger, New York, p.358-400.

MORTATI, A.F. 2004. Colonização por peixes no folhiço submerso: implicações das mudanças na cobertura florestal sobre a dinâmica da ictiofauna de igarapé de terra firme, na Amazônia Central. Dissertação de Mestrado, Instituto Nacional de Pesquisas da Amazônia/Universidade Federal do Amazonas, Manaus.

NEMETH, R.S. 1998. The effect of natural variation in substrate architecture on the survival of juvenile bicolor damselfish. Environ. Biol. Fishes. 53: $129-141$.

PASSOS, M.I.S., NESSIMIAN, J.L. \& DORVILLÉ, L.F.M. 2003. Distribuição espaço-temporal da comunidade de Elmidae (Coleoptera) em um rio da Floresta da Tijuca, Rio de Janeiro, R.J. Bol. Mus. Nac. 509: 1-9.
PENNAK, R.W. 1978. Freshwater invertebrates of the United States. 2 ed. Jonh Wiley \& Sons, New York.

SALCEDO, N.J. \& FONSECA, M.A. 1998. Caracterización de la comunidad bentónica de insectos acuáticos en dos biotopos de un igarapé de la Reserva Ducke, Manaus, Brasil. In Livro do curso de campo de Ecologia da Floresta Amazônica (Venticinque, E.M., ed.). Projeto de Dinâmicas Biológicas de Fragmentos Florestais/ Instituto Nacional de pesquisas da Amazônia, Manaus. http://pdbff.inpa.gov.br/efalivros.html (último acesso em 31/10/2006).

SCULliON, J., PARISH, C.A., MORGAN, N. \& EDWARDS, R.W. 1982. Comparison of benthic macroinvertebrate fauna and substratum composition in riffles and pools in the impounded River Elan and the unreguladed River Wye, mid-Wales. Freshw. Biol. 12: 579-595.

UIEDA, V.S. \& GAJARDO, I.C.S.M. 1996. Macroinvertebrados perifíticos encontrados em poções e corredeiras de um riacho. Naturalia. 21: 31-47.

VANNOTE, R.L., MINSHAL, G.W., CUMMINS, K.W, SEDELL, J.R., \& CUSHING, C.E, 1980. The river continuum concept. Can. J. Fish. Aquat. Sci. 37: 130-137.

ZAR, J. H. 1999. Biostatistical analysis. 4 ed. Prentice-Hall, New Jersey. 